\title{
Leadership in Organisationen sozialer Bewegungen: Kollektive Reflexion und Regeln als Basis für Selbststeuerung
}

\author{
Ruth Simsa ${ }^{1}$ iD \\ Online publiziert: 25 . Juli 2019 \\ (c) Der/die Autor(en) 2019
}

\section{Zusammenfassung}

Dieser Beitrag in der Zeitschrift Gruppe. Interaktion. Organisation analysiert Leadership in Organisationen der spanischen Protestbewegung. Es werden Idealvorstellungen der AktivistInnen von Führung, deren Umsetzung in der Praxis, damit einhergehende Probleme und der Umgang mit diesen Problemen dargestellt. Theoretische Grundlage sind Critical Leadership Studies, die Führung nicht als das Handeln einzelner Personen, sondern als Prozess des gesamten beteiligten Systems interpretieren und damit klar zwischen Leadership und Führungspersonen unterscheiden. Ferner werden Konsequenzen für die Führungspraxis auch in konventionellen Organisationen diskutiert.

Schlüsselwörter Leadership · Bewegungsorganisationen · Critical Leadership Studies

\section{Leadership in social movement organizations}

\begin{abstract}
This paper in the journal Gruppe. Interaktion. Organisation analyses leadership in organizations of the Spanish protest movement. It presents the ideal ideas of the activists of leadership, their implementation in practice, the associated problems and how to deal with these problems. Theoretical basis are Critical Leadership Studies, which interpret leadership not as the actions of individuals, but as the process of the entire system involved and thus clearly distinguish between leadership and leaders. Furthermore, consequences for leadership practice are also discussed in conventional organizations.
\end{abstract}

Keywords Leadership · Social Movement Organizations · Critical Leadership Studies

\section{Einleitung}

„Ich glaube an die Organisation der Gesellschaft von unten, an die Selbstverwaltung, an die direkte Demokratie, an die Ablehnung von Führungspositionen." So formulierte es ein Aktivist der Spanischen Protestbewegung - im Einklang mit vielen anderen Befragten. Was bedeutet dies für Leadership in den Organisationen der Bewegung? Verändern Organisationen, die die Welt verändern wollen, auch ihre Führung? Und was können wir daraus allgemein über Führung lernen? Soziale Bewegungen werden meist nicht gerade mit Führung assoziiert, zeichnen sie sich doch durch Spontaneität, Partizipation und Basisdemokratie aus. Ih-

Ruth Simsa

ruth.simsa@wu.ac.at

1 Wirtschaftsuniversität Wien, Wien, Österreich nen wird daher vorgeworfen, sie würden das Potenzial von Leadership nicht für effektives Organisieren nutzen (Western 2014). Dies stimmt gegenwärtig allerdings nicht mehr, schon gar nicht, wenn man zwischen sozialen Bewegungen und sozialen Bewegungsorganisationen $\left(\mathrm{SMO}^{1}\right)$ unterscheidet. Hier gibt es nicht nur weithin geteilte, sondern auch sehr elaborierte Vorstellungen passender bzw. gewünschter Führung. Zwischen verschiedenen Bewegungen wie dem Alterglobalization Movement oder Occupy werden theoretische Überlegungen zum Thema Führung ebenso ausgetauscht, wie praktische Anleitungen, das Thema Leadership wird als wichtig erkannt, es wird damit experimentiert und Methoden wie Theorien werden weiterentwickelt.

Im Folgenden soll anhand einer empirischen Untersuchung in Spanien gezeigt werden, welche Führung Akti-

${ }^{1}$ Die Abkürzung SMO für Social Movement Organizations hat sich durchgesetzt und wird daher auch hier verwendet. 
vistInnen anstreben, um Partizipation und Demokratie im Organisationsalltag zu verankern. Leadership wird hier definiert als Prozesse und Praktiken, welche Gruppen oder Organisationen Orientierung geben (Crevani 2018, S. 88). Nach einer theoretischen und empirischen Bestandsaufnahme geht es um die Frage, was herkömmliche Organisationen von diesen Bemühungen lernen können. Diese sehen sich zum Teil Ansprüchen an „New Work“, mit demokratischeren, partizipativen Formen der Zusammenarbeit und Führung gegenüber, nicht alle können sich auf die für Junge prekäre Arbeitsmarktlage verlassen und auf hierarchischen Verhältnissen beharren.

\section{Kontext}

Die Protestbewegung Spaniens begann im Jahr 2011 als Reaktion auf massive soziale Probleme infolge der Wirtschaftskrise und der Austeritätspolitik, auf hohe Korruption und auf eine generelle Krise des Vertrauens in das politische System (Benski et al. 2013; Romanos 2017). Nach ihrem Gründungsdatum im Mai wird sie als $15 \mathrm{M}$ bezeichnet. Anders als in vielen Ländern, in denen sich Krisenverlierer rechtspopulistischen Strömungen zuwenden, kam es hier zu starken Solidarisierungseffekten und zu vielfältigen zivilgesellschaftlichen Aktivitäten, die von Protest und politischer Arbeit bis zu engmaschiger sozialer Hilfe und Unterstützung reichen. Das von Crouch attestierte postdemokratische Desinteresse an Politik (Crouch 2004) war in Spanien jedenfalls nicht beobachtbar.

Die Aktivitäten der Bewegung waren vielfältig, neben Protesten, Demonstrationen und Platz- oder Hausbesetzungen entstand ein dichtes Netz von Bewegungsorganisationen, das von der bundesweiten Plattform gegen Delogierungen bis hin zu kleineren Gruppen wie etwa Jugendlichen ohne Zukunft, den Protest-Großeltern, Frauen- oder Anwaltsinitiativen, Stadtteilgruppen, Food-Coops oder Kulturorganisationen reicht. Es werden u. a. Rechtsberatung, Weiterbildung, Zeitbanken, alternative Währungen, oder private Essensausgaben an Hungernde organisiert. Dies alles und in der Folge die Bildung von bundesweiten und regionalen Parteien im Rahmen der Bewegung hatten Auswirkungen auf die politische Situation. So verzeichneten die regierenden Konservativen bei den Wahlen 2015 die schwächsten Ergebnisse seit 1991 (Wandler 2015) und das Zweiparteiensystem, das seit dem Ende der Diktatur 1978 bestanden hatte, wurde gebrochen. Die Bewegung hatte auch Auswirkungen auf die politische Kultur. Nachdem in Spanien lange Zeit eine Tradition des Schweigens, des sich Fernhaltens von Politik und damit von Problemen geherrscht hatte - ein Phänomen, das als „soziologischer Franquismo“ bezeichnet wurde und auch junge Menschen betroffen hatte, die die Diktatur nicht erlebt hatten - wurde Politisierung nun alltäglicher. Ein Aktivist meinte: „Was sich wirklich verändert hat, ist die Art und Weise, wie wir über Politik denken. Wir haben begonnen, über Politik zu sprechen, und wir werden nicht wieder still sein. "Während nach 2011 die Protestaktivitäten stark zunahmen (García 2013), gab es ab ca. 2014 einen Rückgang. Dies ist zum einen mit meist beobachtbaren Protestzyklen erklärbar, zum anderen auch mit deutlich gestiegener Repression (Simsa 2017). Spätestens 2015 kam es zu starken Demobilisierungen bzw. zur Entwicklung einer neuen, stark von Frauen(themen) getriebenen Bewegung. Viele der im Zuge der Bewegung gegründeten Organisationen existieren allerdings weiterhin und sie identifizieren sich zumeist auch noch mit 15M.

\section{Methoden}

Die Arbeit basiert auf qualitativer Forschung. Insgesamt wurden von 2014 bis 2017 neben 16 teilnehmenden Beobachtungen 92 qualitative Interviews mit AktivistInnen und ExpertInnen in Madrid, Sevilla und Valencia durchgeführt. Aus der gesamten Stichprobe wurden 31 für das Forschungsthema besonders geeignete Interviews gewählt, die transkribiert, codiert und ausgewertet wurden. ${ }^{2}$

\section{Theoretische Basis}

Theoretische Grundlage der Analyse sind Critical Leadership Studies, die Führung nicht als das Handeln einzelner Personen, sondern als Prozess des gesamten beteiligten Systems interpretieren und damit klar zwischen Leadership und Führungspersonen unterscheiden (Simsa und Totter 2020; Sutherland et al. 2014). Sie interpretieren Leadership als relationalen, sozial konstruierten Prozess, der das Ergebnis der Interaktionen diverser Akteure ist (Bolden 2011; Gronn 2002). Effekte des Leadership resultieren nicht von einzelnen Individuen, sondern von der Dynamik im spezifischen System. Damit theoretisieren Critical Leadership Studies das, was Aktivisten in die Praxis umsetzen wollen, nämlich einen Fokus auf Prozesse und Beziehungen, statt auf Führungspositionen. Aus einem gänzlich anderen Kontext heraus kommen sie damit zu ähnlichen Schlüssen wie die Systemtheorie und zum Teil auch die Gruppendynamik. Ein zentraler Aspekt ist die Frage, wie organisationale Klarheit geschaffen werden kann ohne Macht an einzelne Individuen zu geben, mittels einer klareren Unterscheidung von Leadership und Leaders, entsprechend der alten anarchistischen Idee das ,Anarchy is not without leadership, it is without followership“ (Ehrlich 1979, p. 108).

\footnotetext{
2 Vielen Dank an Marion Totter für die Mitarbeit bei der Codierung und der Interpretation der Ergebnisse.
} 
SMOs älterer sozialer Bewegungen, wie z.B. der 68er Bewegung, wird nachgesagt, sie wollten zuerst die Welt und dann erst ihre eigenen Organisationen verbessern. Im Unterschied dazu verstehen sich Organisationen der heutigen autonomen Bewegungen als präfigurativ (Simsa und Totter 2017; Yates 2014), d.h. sie haben den Anspruch, gesellschaftliche Ziele im Hier und Jetzt der Organisationen umzusetzen. Viele Akteure beziehen sich in ihrer Identität und ihren Zielen explizit auf neue Formen von Organisation. Damit einher gehen auch spezifische Formen des Leadership.

Es gibt relative wenige empirische Studien zum Leadership in SMOs - dies könnte eine Art Gegenübertragung des Forschungsfeldes, welches sich lange zu Leadership distanziert gezeigt hatte, auf die Forschung darstellen. Einzelne Artikeln fokussieren auf interne (Entscheidungs)strukturen (Della Porta et al. 2009; Graeber 2012; Leach 2009; Polletta 2005), andere auf Meetings (Haug 2011, 2013; Thorburn 2012) oder Konflikte (Laamanen und Den Hond 2015; Maeckelbergh 2012). Andere AutorInnen zeigen die Risiken von fehlender Führung auf, wie zum Beispiel Ineffizienz oder informelle Machtstrukturen (Freeman 1970; Polletta 2002; Vecchio et al. 2010).

Generell wird in der Literatur über Leadership in sozialen Bewegungen meist argumentiert, dass deren Organisationen zu nicht-hierarchischen Formen des Organisierens tendieren und direkte, partizipative Demokratie bzw. Autonomie umsetzen wollen (Benski et al. 2013; Polletta 2002). Prinzipien von Selbstorganisation und anarchistischem Denken sind demnach oft handlungsleitend (Bratich 2007; Gibson 2013; Graeber 2011), wie die Ablehnung von Autorität, Hierarchie und jeglicher Dominanz (Wigger 2014, S. 743). Die Bewegungen werden daher als autonom charakterisiert (Flesher Fominaya 2015, S. 145). Ihre Prinzipien sind weitgehend Selbststeuerung und Egalität, Partizipation, Solidarität und Inklusion (Simsa 2016).

\section{Leadership in den sozialen Bewegungsorganisationen (SMO): Führung, aber kaum Führungspositionen}

\footnotetext{
„Wir haben eine Philosophie der Führung, nicht der Führer, vielmehr der beteiligten Personen, die selbst Entscheidungen treffen". Während die soziale Bewegung Leadership grundsätzlich vermeiden will, sind sich VertreterInnen der Organisationen der Notwendigkeit von Leadership durchaus bewusst. Führungspositionen werden abgelehnt, nicht aber Führung. Aktivisten teilen ziemlich genaue Ansichten darüber, was gute Führung für sie bedeutet.

Zentral in fast allen Interviews ist der Begriff der Selbststeuerung (autogestión). Leadership darf demnach keine Form von Hierarchie, formaler Autorität oder festen Rollen
}

implizieren, es muss die gleichberechtigte Beteiligung aller Mitglieder ermöglichen und transparent sein. Formale wie auch informelle Leadership-Positionen, deren Inhaber Entscheidungsmacht haben, werden abgelehnt, Führungsarbeit von Einzelpersonen aber geschätzt, sofern sie den Idealen der Autonomie entspricht und nicht als hierarchisches topdown-Handeln wahrgenommen wird. In der Regel bedeutet dies die nur vorübergehende Übernahme von Führungspositionen durch Einzelpersonen (z.B. der Moderator eines Meetings) und nur dann, wenn dies als im Dienst des Kollektivs gesehen wird und permanent durch dieses legitimiert wird. Nur in Ausnahmefällen entstehen stabilere Positionen (z.B. die langjährige Sprecherin der landesweiten Organisation gegen Zwangsräumungen, die gegenwärtige Bürgermeisterin von Barcelona).

Austauschbarkeit von Personen und die Fähigkeit, als Kollektiv zu handeln, werden angestrebt. ,Wir haben eine Führungsphilosophie, und das sind keine Bosse, sondern Menschen, die sich engagieren, die handeln und die am Ende die anderen ermächtigen“. Das Ideal konsensueller Entscheidungsfindung herrscht vor, nur in Ausnahmefällen werden Mehrheitsentscheidungen angewandt.

Ein Beispiel ist die 2011 gegründete Organisation „Legal Sol", eine Gruppe von Anwälten, die neben politischer Arbeit, wie Aktivismus gegen repressive Gesetze, Rechtsaufklärung und der Anklage von Korruption auch Rechtsvertretung für angeklagte AktivistInnen durchführt. Zwischen 2011 und 2015 hat sie 493 Prozesse verhandelt und nur 6\% davon verloren. Die Organisation leistet hochprofessionelle Arbeit in einem sehr spezialisierten Feld, ist weithin bekannt und von AktivistInnen geschätzt. Dennoch unterscheiden sich ihre Arbeits- und Organisationsformen stark von den traditionellen Kanzleien: Die Mitgliedschaft ist nur geringfügig eingeschränkt, die Organisation ist sehr informell, Führung und Verantwortung für Kommunikation, Dokumentation und Repräsentation rotieren, und viel Zeit wird den persönlichen Bedürfnissen der Mitglieder gewidmet. Doch in der scheinbar chaotischen Struktur gibt es sehr klare Regeln, vor allem in Bezug auf Verantwortlichkeiten, Kommunikationsstile und Rahmenbedingungen. In zwei Vorfällen der partizipativen Beobachtung konnten der Wechsel der Führung in Bezug auf bestimmte Themen, der sorgfältige Umgang mit Konflikten und Ritualen wie ritualisierte Rückmeldungen an die Moderation beobachtet werden.

\section{Herausforderungen in der Praxis}

Nicht überraschend impliziert dieses Ideal der autonomen Führung gewisse Schwierigkeiten in der Praxis, insbesondere informelle Hierarchien und Ineffizienzen. Informelle Hierarchien entstehen durch unterschiedliche Fähigkei- 
ten und Ressourcen (Zeit, Rhetorik), durch Bonding-Effekte (Kerngruppe), durch gesellschaftliche Machtstrukturen (Frauen vs. Männer) etc.: „Selbst innerhalb einer Versammlung, auch wenn das ein Raum der Gemeinschaft ist, sind diejenigen, die am meisten sprechen, die laut sprechen, die Männer ...". Es wird auch von subtilen Formen der Manipulation, etwa durch Agenda-Setting und Einflussnahme auf Diskussionen, berichtet.

Ineffizienzen entstehen durch die Ideale der Offenheit, Rotation und der breiten Partizipation, hier gibt es einen Trade-off: „Zwei politische Strukturen waren schon immer kontroversiell: die Wirksamkeit der Auswahl einer Führungsgruppe und (...) die Langsamkeit, aber mehr Partizipation und Demokratie, wenn alles auf den Versammlungen basiert." Zwar wurden in den Organisationen gewisse Zugangsregeln entwickelt, dennoch sind Entscheidungsprozesse oft langwierig. Während die egalitären Prozesse im „Alltagsgeschäft“, eher gut funktionieren, kommt es v.a. in Zusammenhang mit strategischen Änderungen zu Problemen: „Das Problem ist, wenn Du Strategien ändern willst oder wenn man ein Ziel erreicht hat. Mit dieser Organisation ist es sehr schwierig, neue Ziele zu entwickeln, wer sollte darüber entscheiden?"

\section{Kollektive Reflexion und Regeln}

Die genannten Probleme sind bekannt aus älteren Arbeiten zu basisdemokratischer Organisation (Krainz und Simsa 1994). Interessant allerdings und m.E. mit neuer Qualität sind Umgangsformen mit diesen Herausforderungen. Aus der Erhebung gehen klar zwei weit geteilte Praktiken hervor, nämlich kollektive Reflexion sowie geteilte, klare und oft strikte Regeln. Beides wird häufig ,Methodologie der Organisation" genannt.

Die Reflexion interner Prozesse wird als Teil der täglichen Arbeit beschrieben. Nachdem Organisationen als präfigurativ verstanden werden, gibt es eine hohe Bereitschaft zu Experimenten mit organisationalen Formen und Prozessen. Dies impliziert auch die permanente Überprüfung, Diskussion und Weiterentwicklung von Führungspraktiken. AktivistInnen betonen die Notwendigkeit kollektiver Reflexion, um organisationale Dynamiken zu verstehen, gemeinsam zu lernen und effiziente Führungspraktiken zu entwickeln. Das theoretische Verständnis von Organisation und Führung sowie die Prozess- und Methodenkompetenz scheinen höher ausgeprägt zu sein als in Wirtschaftsorganisationen. Die Bereitschaft, sich kollektiver Beobachtung, Rückmeldung und Reflexion auszusetzen, wird in Zusammenhang mit guter Leadership-Arbeit gebracht. Wie auch in klassischen Beratungsprozessen, sind die konkreten Praktiken dieser Reflexion zwar bisweilen vom Ergebnis her, nicht aber der Form nach spektakulär. In vielen SMOs et- wa gibt es diesbezügliche Routinen, etwa am Ende jeder Besprechung eine kurze Feedbackrunde an den Moderator. Leadership wird explizit thematisiert. Auch organisationsübergreifende Trainings über Methodologien werden durchgeführt.

Interessanterweise werden reflexive Kompetenzen in der Literatur fast ausschließlich als notwendige Fähigkeiten individueller Leader beschrieben (Day 2001). Auf organisationaler Ebene sind Reflexion und damit korrespondierende Leadership-Praktiken kaum Thema empirischer Forschung.

Die hohe Bedeutung von Regeln ist bemerkenswert, da Bewegungen und ihre Organisationen in der Regel als besonders spontan gesehen werden. Regeln haben aber - wie generell in anarchistischen Konzepten - hohe Bedeutung, insbesondere in Bezug auf die Verhinderung informeller Hierarchien. Sie sind häufig Gegenstand von Konflikten, teils explizit formuliert, teils implizit aber nichtsdestotrotz hochbindend. Neben häufig allgemeinen Regeln wie etwa, andere nicht zu dominieren, gibt es SMOs mit sehr detailreich formulierten Verhaltensregeln, die ,gute Kommunikation" garantieren sollen. Auch hier wird mit Methoden experimentiert, etwa unterschiedliche Karten, die jeweils eine bestimmte Redezeit verkörpern und nach jeder Wortmeldung abgegeben werden müssen, Reißverschlusssysteme für Wortmeldungen von Männern und Frauen, geschlossene Runden, in denen zu einem Thema nur eine bestimmte Anzahl von Personen sprechen darf, etc.: „Das funktioniert. Es scheint fast magisch."

Oft sind Umgangsformen und Verhaltensregeln allerdings implizit geregelt: „Was uns am besten gefällt, ist, dass wir kein Dokument haben, das unsere diskursiven Strategien erklärt; es ist nicht notwendig, weil wir - aus dem einen oder anderen Grund - eine bestimmte Sprache verinnerlicht haben (...) wir sind nicht monolithisch, aber von außen erscheint eine gewisse Kohäsion. Das resultiert aus der täglichen Arbeit." Dies mag Vor- und Nachteile haben. Regeln werden jedenfalls als ebenbürtig, wenngleich in einem dialektischen Verhältnis zu Autonomie und Spontaneität gesehen.

Bemerkenswert sind auch die hohe Bereitschaft, ProzessKnow How zu entwickeln, sowie die oft hohen Kommunikations- und Prozesskompetenzen, die mit der Entwicklung von geeigneten Regeln und mit kollektiver Reflexion einhergehen. Seit der Veröffentlichung von Artikeln wie „,The tyranny of structurelessness“ (Freeman 1970) und „Freedom is an endless meeting“ (Polletta 2002), die die praktischen Probleme der egalitären Organisation betonten, haben AktivistInnen von SMO dazugelernt. Während critical leadership studies das emanzipatorische Potenzial von Führung möglicherweise unterschätzen (Alvesson und Spicer 2012), tun dies die AktivistInnen nicht. Sie haben klare Vorstellungen davon, wie Führungsarbeit umgesetzt werden 
sollte, und sind bereit, in deren Entwicklung und alltägliche Umsetzung zu investieren.

\section{Konsequenzen für die Praxis konventioneller Organisationen}

Praktiken der Bewegungsorganisationen sind vermutlich nicht auf Organisationen in Wirtschaft oder Verwaltung zu übertragen, zu verschieden sind Ziele, Ideologien und Rahmenbedingungen. Auch die Frage der Skalierbarkeit ist offen. Nicht umsonst erschöpfen sich die in regelmäßigen Abständen - derzeit v.a. unter dem Label „Agil“ - proklamierten Modelle der Etablierung egalitärer Strukturen und des „Leadership ohne Leader“ oft in rhetorischen Bekenntnissen oder in der Gestaltung kleiner Teams. Die Sehnsucht nach neuen Formen ist jedenfalls vorhanden. Dies zeigt die lange Geschichte der, oft als Fortschrittsframe von strikter Hierarchie zu aufgeklärter Selbstorganisation konstruierten, enthusiastisch vertretenen Versuche, die von teilautonomen Gruppen der 1920er-Jahre, über die in den 1950er-Jahren entwickelte Soziokratie, Holacracy der 2000er-Jahre und der gegenwärtigen agilen Organisation reichen. Und angesichts der Nachteile der Hierarchie (Heintel und Krainz 2011) ist wohl auch die Notwendigkeit von klugen und neuen Konzepten von Führung gegeben.

Angesichts der nicht linear kontrollierbaren Eigendynamiken von Organisationen wird in der Theorie schon lange reflexive Steuerung empfohlen (Krainz 2011). Es wird argumentiert, dass die Komplexität und Dynamik der Wirtschaft eine beweglichere und partizipativere Gestaltung der Führung erfordern (Buchinger und Schober 2006), und dass die Förderung von individuellen LeistungsträgerInnen nicht automatisch auch organisationale Kompetenzen im Umgang mit Herausforderungen fördert (Wimmer und Schumacher 2009), und dass es einzelnen Führungskräften nicht gelingen kann, mittels individuellen Bemühens strukturelle Probleme der Organisation auszugleichen (Simsa 2015). In der Praxis der meisten Organisationen ist dies allerdings noch nicht angekommen, gerade in Krisensituationen wird hier immer noch auf Hierarchie und starke Führer gesetzt.

Dennoch können aus den Beobachtungen der SMOs Anregungen formuliert werden, die mit der Logik von konventionellen Organisationen vereinbar sein sollten.

Erstens: Etabliere Zeit für regelmäßige kollektive Reflexion: Dies muss sich nicht nur im Rahmen jährlicher Strategie- oder Teamworkshops erschöpfen. Im Rahmen kollektiver Selbstaufklärung geht es darum, die eigene Organisation und ihre Dynamik zu verstehen, sie auf Angemessenheit zu überprüfen und experimentell dort zu verändern, wo Prozesse dysfunktional sind. Dynamiken der Führung sind eng mit jenen der Organisation verbunden, jegliche Versuche, Führung neu zu gestalten, müssen daher an der Gestaltung der Organisation ansetzen. Mit ihrer Aufmerksamkeit für Prozesse, Experimenten mit der eigenen Organisation und mit kollektiver Reflexion praktizieren die AkteurInnen der SMOs das, was Organisationsentwicklung und Gruppendynamik schon lange (und oft vergeblich) im Alltag konventioneller Organisationen verankern wollen.

Zweitens: Nutze diese kollektive Selbstaufklärung auch für die gemeinsame Entwicklung und Überprüfung von handlungsleitenden Regeln. Dies betrifft nicht nur formale Regelwerke, damit sind die meisten Organisationen gut ausgestattet, sondern auch die weicheren, oft informellen und die auf Verhalten bezogenen Regeln. Nicht ohne Grund wird im Rahmen von extern begleiteten Strategieworkshops häufig das Bedürfnis geäußert, Verhaltensrichtlinien zu diskutieren und zu formulieren. Wie soll Kooperation gestaltet werden, wie mit Fehlern umgegangen, mit Konflikten, etc.?

Drittens: Unterziehe auch die gelebte Führung kollektiver Reflexion. Selbst, wenn Führungspositionen nicht abgeschafft werden sollen, kann gemeinsam hinterfragt werden, wie weit die Gestaltung von Führung der Organisation und Situation angemessen ist. Aus der Beratung wissen wir, dass regelmäßiges Feed-back die Qualität des Führungshandelns erhöhen kann und dass verteilte Führung bzw. Führungskompetenz zu höherer organisationaler Problemlösungskompetenz führen können.

AkteurInnen in SMOs gehen davon aus, dass gesellschaftliche Ziele wie Demokratie oder Partizipation in der alltäglichen Gestaltung von Organisationen und von Führung gelebt werden müssen. Sie sind bereit, in die Entwicklung neuer Formen und Methoden zu investieren - so unvollkommen ihre Umsetzung manchmal auch sein mag. Sie fokussieren dabei auf adäquate Prozesse und Führungshandeln anstatt auf Positionen. Und sie haben den Mut, das Führungshandeln der regelmäßigen kollektiven Reflexion und des Feed-Backs zu unterziehen. Die hohe Bedeutung von Führung wird in allen Organisationen erkannt. Die Antworten auf diese Diagnose sind in den einzelnen Feldern hoch verschieden, jene der AktivistInnen könnten allerdings auch AkteurInnen in konventionellen Organisationen inspirieren.

Funding Open access funding provided by Vienna University of Economics and Business (WU).

Open Access Dieser Artikel wird unter der Creative Commons Namensnennung 4.0 International Lizenz (http://creativecommons.org/ licenses/by/4.0/deed.de) veröffentlicht, welche die Nutzung, Vervielfältigung, Bearbeitung, Verbreitung und Wiedergabe in jeglichem Medium und Format erlaubt, sofern Sie den/die ursprünglichen Autor(en) und die Quelle ordnungsgemäß nennen, einen Link zur Creative Commons Lizenz beifügen und angeben, ob Änderungen vorgenommen wurden. 


\section{Literatur}

Alvesson, M., \& Spicer, A. (2012). Critical leadership studies: The case for critical performativity. Human Relations, 65(3), 367-390.

Benski, T., Langman, L., Perugorría, I., \& Tejerina, B. (2013). From the streets and squares to social movement studies: what have we learned? Current Sociology, 61(4), 541-561. https://doi.org/10.1177/ 0011392113479753.

Bolden, R. (2011). Distributed leadership in organizations: a review of theory and research. International Journal of Management Reviews, 13(3), 251-269. https://doi.org/10.1111/j.1468-2370.2011. 00306.x.

Bratich, J. (2007). A review of: "Gramsci is dead: anarchist currents in the newest social movements by R. Day". The Communication Review, 10(2), 167-170. https://doi.org/10.1080/ 10714420701350445.

Buchinger, K., \& Schober, H. (2006). Das Odysseusprinzip. Leadership revisited. Stuttgart: Klett-Cotta.

Crevani, L. (2018). Is there leadership in a fluid world? Exploring the ongoing production of direction in organizing. Leadership, 14(1), 83-109. https://doi.org/10.1177/1742715015616667.

Crouch, C. (2004). Post-democracy. Polity: Cambridge.

Day, D. V. (2001). Leadership development: A review in context. The Leadership Quarterly, 11(4), 581-613.

Ehrlich, H.J. (1979). Reinventing anarchy, again. AK Press: Edinburgh.

Flesher Fominaya, C. (2015). Debunking spontaneity: Spain's 15-M/ Indignados as autonomous movement. Social Movement Studies, 14(2), 142-163. https://doi.org/10.1080/14742837.2014.945075.

Freeman, J. (1970). The tyranny of structurelessness. http://www. democraciarealya.es/manifiesto-comun/manifesto-english/. $\mathrm{Zu}$ gegriffen: 12. Okt. 2017.

García, O. J. M. (2013). Soft repression and the current wave of social mobilisations in Spain. Social Movement Studies, 13(2), 303-308. https://doi.org/10.1080/14742837.2013.863147.

Gibson, M.R. (2013). The anarchism of the occupy movement. Australian Journal of Political Science, 48(3), 335-348. https://doi. org/10.1080/10361146.2013.820687.

Graeber, D. (2011). Occupy wall street's anarchist roots. http://www. aljazeera.com/indepth/opinion/2011/11/2011112872835904508. html. Zugegriffen: 31. Mai 2016.

Graeber, D. (2012). Inside occupy. Frankfurt, New York: Campus.

Gronn, P. (2002). Distributed leadership as a unit of analysis. The Leadership Quarterly, 13(4), 423-451. https://doi.org/10.1016/ S1048-9843(02)00120-0.

Haug, C. (2011). The construction of consensus in social movement meetings: mechanisms and practices of face-to-face decisionmaking. Paper presented at the European Group of Organizational Studies.

Haug, C. (2013). Organizing spaces: meeting arenas as a social movement infrastructure between organization, network, and institution. Organization Studies, 34(5/6), 705-732. https://doi.org/10. 1177/0170840613479232.

Heintel, P., \& Krainz, E.E. (2011). Projektmanagement. Hierarchiekrise, Systemabwehr, Komplexitätsbewältigung (5. Aufl.). Wiesbaden: Gabler.

Krainz, E.E. (2011). Leiden an der Organisation. In K. M. Ratheiser, J. Menschik-Bendele, E. E. Krainz \& M. Burger (Hrsg.), Burnout und Prävention. Ein Lesebuch für Ärzte, Pfleger und Therapeuten. Wien, New York: Springer.

Krainz, E.E., \& Simsa, R. (1994). Gute Menschen. Beratung in Freiwilligen-Organisationen. In R. Grossmann, E. Krainz \& M. Oswald (Hrsg.), Veränderung in Organisationen. Wiesbaden: Gabler.

Laamanen, M., \& Den Hond, F. (2015). Prefigurative partial organization in local social movements: examining decided and emergent order in a time bank. Paper presented at the EGOS, Athens.
Leach, D. K. (2009). An elusive "we": Antidogmatism, democratic practice, and the contradictory identity of the German Autonomen. American Behavioral Scientist, 52(7), 1042-1068. https:// doi.org/10.1177/0002764208327674.

Maeckelbergh, M. (2012). Horizontal Democracy Now: From Alterglobalization to Occupation. Interface, 4(1), 207-234.

Polletta, F. (2002). Freedom is an endless meeting: democracy in American social movements. Chicago: University of Chicago Press.

Polletta, F. (2005). How participatory democracy became white: Culture and organizational choice. Mobilization: An International Quarterly, 10(2), 271-288.

Porta, D.D., Kriesi, H., \& Rucht, D. (2009). Social Movements in a Globalizing World. Basingstoke: Palgrave Macmillan.

Romanos, E. (2017). Late Neoliberalism and its indignados: contention in austerity Spain. In D. Della Porta, M. Andretta, T. Fernandes, F. O'Connor, E. Romanos \& M. Vogiatzoglou (Hrsg.), Late Neoliberalism and its discontents in the economic crisis: comparing social movements in the European periphery (S. 131-167). Cham: Springer.

Simsa, R. (2015). Perspektive systemische Beratung. In M. Patak \& R. Simsa (Hrsg.), Perspektive systemische Beratung (S. 153-163). Wien: Linde.

Simsa, R. (2016). Arbeit außerhalb der Arbeitswelt - neue Organisationen im Rahmen sozialer Bewegungen am Beispiel Spaniens. Gruppe. Interaktion. Organisation, 47(1), 53-60. https://doi.org/ 10.1007/s11612-016-0309-7.

Simsa, R. (2017). Repression of the Spanish protest movement - mechanisms and consequences. Nonprofit Policy Forum, 8(3), 321-336. https://doi.org/10.1515/npf-2017-0022.

Simsa, R., \& Totter, M. (2017). Social Movement Organizations in Spain - Being Partial as the Prefigurative Enactment of Social Change. Qualitative Research in Organizations and Management, 12(4), 280-296. https://doi.org/10.1108/QROM-01-2017-1470.

Simsa, R., \& Totter, M. (2020). Leadership in Social Movement Organizations. Ephemera, forthcoming.

Sutherland, N., Land, C., \& Böhm, S. (2014). Anti-leaders(hip) in social movement organizations: the case of autonomous grassroots groups. Organization, 21(6), 759-781. https://doi.org/10.1177/ 1350508413480254.

Thorburn, E.D. (2012). A common assembly: multitude, assemblies, and a new politics of the common. Interface: A Journal For and About Social Movements, 4(2), 254-279.

Vecchio, R.P., Justin, J.E., \& Pearce, C.L. (2010). Empowering leadership: An examination of mediating mechanisms within a hierarchical structure. The Leadership Quarterly, 21(3), 530-542.

Wandler, R. (2015). Die Empörten an der Macht in Spanien. http:// derstandard.at/2000017437267/Die-Empoerten-an-der-Machtin-Spanien. Zugegriffen: 14. Juni 2017.

Western, S. (2014). Autonomist leadership in leaderless movements: Anarchists leading the way. ephemera. theory and politics in organization, 14(4), 673-698.

Wigger, A. (2014). A critical appraisal of what could be an anarchist political economy. Ephemera, 14(4), 739.

Wimmer, R., \& Schumacher, T. (2009). Führung und Organisation. In R. Wimmer, J. O. Meissner \& P. Wolf (Hrsg.), Praktische Organisationswissenschaft. Lehrbuch für Studium und Beruf (S. 217-240). Heidelberg: Carl Auer.

Yates, L. (2014). Rethinking prefiguration: alternatives, micropolitics and goals in social movements. Social Movement Studies: Journal of Social, Cultural and Political Protest, 14(1), 1-21. 


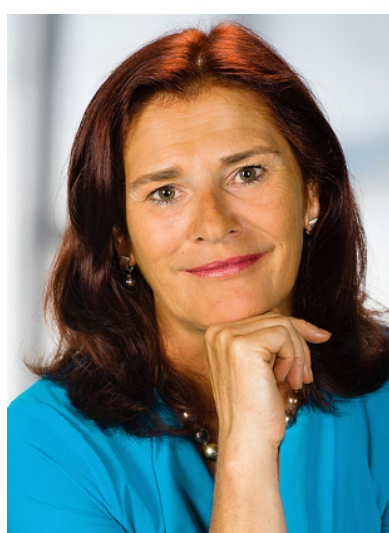

Ruth Simsa a.o. Univ. Prof. am Institut für Soziologie und Empirische Sozialforschung der Wirtschaftsuniversität Wien. Vorstand des Instituts für Nonprofit-Organisationen, WU-Wien; www.npo.or.at; Organisationsberaterin und Führungskräftetrainerin (www.ruthsimsa.at). Editor-in-Chief: VOLUNTAS International Journal of Voluntary and Nonprofit Organizations. 